\section{Endoscopic advanced imaging of the respiratory tract: exploring probe-based confocal laser endomicroscopy in emphysema}

\section{ABSTRACT}

Probe-based confocal laser endomicroscopy ( $p C L E)$ was performed in 15 patients with emphysema and 15 healthy subjects to visualise small airways in a direct and dynamic way. Morphometry shows that the median cross-sectional area of the alveolar openings at the level of the alveolar ducts is significantly larger in emphysema $\left(7.2 \times 10^{4} \mu \mathrm{m}^{2}\right)$ as compared with healthy subjects $\left(5.2 \times 10^{4} \mu \mathrm{m}^{2}\right)(p=0.0002)$. Normalised autofluorescence intensity histograms show a decrease in median autofluorescence intensity (mAFI) in emphysema $(p=0.001)$. mAFI correlates well with Tiffeneau index $(r=0.66$, $\mathrm{p}=0.007,95 \% \mathrm{Cl} 0.21$ to 0.88 ).

Autofluorescence intensity in emphysema correlates with corresponding data of CT-based quantification. pCLE-based morphometry and autofluorescence intensity analysis in emphysema is able to detect regional changes inside the 'quiet zone'.

Trial registration number Results, NCT01204970.

\section{INTRODUCTION}

The local architecture and the bioenvironment of small airways in emphysema and their relation to disease heterogeneity are yet to be explored. Probe-based confocal laser endomicroscopy (pCLE) is an endoscopic imaging technique using a semiflexible probe that can be guided through the human airways into the periphery of the lung. The optical images that are generated mainly depend on the autofluorescence characteristics of elastic fibres situated throughout the tracheobronchial wall $^{1}$ and at the level of the pulmonary acinus. $^{2}$ This study assesses structural changes in emphysema detected by pCLE and the possible correlation with lung function and quantitative CT.

\section{MATERIAL AND METHODS}

pCLE imaging was obtained using a standardised protocol. Postprocedural analysis was performed in a frame-by-frame method, and frames were selected for quantification based upon image quality (figure 1). The remaining image stack was quantified for morphological descriptors (see online supplementary figure S1) and for fluorescence intensity. Detailed methodology can be found in online supplementary panel S1.

\section{RESULTS}

Patient characteristics are listed in the online supplementary table S1.

Values for the longest diameter of the alveolar openings at the level of the alveolar ducts (LDAD), the median crosssectional area of alveolar duct opening (ADA) and median elastic fibre thickness (EFT) are shown in table 1. The IQRs for individual measurements of LDAD, ADA and EFT within a specific subject (as a measure for within-subject variability) are presented in table 1.

Normalised autofluorescence intensity (AFI) histograms were quantified by three variables: median autofluorescence intensity (mAFI), the autofluorescence intensity marking the fifteenth percentile (fAFI) and the proportional amount of pixels characterised by an AFI lower than 100 (PA100). Mean values for AFI in healthy subjects and for emphysema can be found in table 1 . The difference in total lung scores for AFI differed significantly between emphysema and healthy subjects ( $p$ values can be found in table 1). AFI histograms were calculated on a lobar level for patients with emphysema. Mean values for mAFI, fAFI and PA100 for upper and lower lobes respectively can be found in table 1 . The difference in AFI between upper and lower lobes in emphysema did not reach statistical significance.
CT density calculation for emphysema based on the average value for lung mean density (LMD), the average value for the fAFI of pixel density (15P) and the average value for the proportion of low attenuation areas with density less than -950 HU (Hounsfield units) (LAA-950) can be found in table 1 . The difference between upper and lower lobes did not reach statistical significance.

A complete correlation matrix for AFI and CT density can be found in the online supplementary table S2. We found the strongest statistical correlations between mAFI and $15 \mathrm{P}(\mathrm{r}=0.56, \mathrm{p}=0.007,95 \% \mathrm{CI}$ 0.16 to 0.80$)$, fAFI and $15 \mathrm{P}(\mathrm{r}=0.62$, $\mathrm{p}=0.001,95 \%$ CI 0.28 to 0.82$)$ and between PA100 and LAA-950 $(\mathrm{r}=0.58$, $\mathrm{p}=0.02,95 \%$ CI 0.12 to 0.84 ). IQR ADA, as a measure for morphological variability within a patient, correlates with $15 \mathrm{P}(\mathrm{r}=$ $-0.67, \mathrm{p}=0.005,95 \% \mathrm{CI}-0.88$ to -0.25$)$ and with $\operatorname{LMD}(\mathrm{r}=-0.47, \mathrm{p}=0.05,95 \%$ $\mathrm{CI}-0.78$ to -0.03$)$. Although not statistically significant, we noted a correlation between mAFI and lobar volume, as calculated by CT segmentation $(\mathrm{r}=-0.38$, $\mathrm{p}=0.09,95 \% \mathrm{CI}-0.76$ to 0.09 ).

A complete correlation matrix for AFI and lung functional data can be found in the online supplementary table S3. mAFI correlates well with the Tiffeneau index $(\mathrm{r}=0.66, \mathrm{p}=0.007,95 \%$ CI 0.21 to 0.88$)$. There is a good correlation between fAFI

Figure 1 Alveoloscopic image showing a ring built out of elastic fibres at the level of an alveolar opening into an alveolar duct (transversal plane). The measurements for the longest internal diameter of the alveolar duct opening or longest diameter of the alveolar openings at the level of the alveolar ducts (yellow line), the perpendicular short axis or 'SDAD' (green line), the elastic fibre thickness (blue line), the diameter of alveolar cells (red line) and the autofluorescence of alveolar cells (pink line) are shown. SDAD, perpendicular short axis. 
Table 1 Data on morphometry, autofluorescence intensity, CT density and their variability

\begin{tabular}{|c|c|c|c|}
\hline & Healthy & Emphysema & Significance \\
\hline \multicolumn{4}{|c|}{ Median values per group } \\
\hline \multicolumn{4}{|c|}{ LDAD in $\mu \mathrm{m}$ (IQR) } \\
\hline Total & $297(244-357)$ & $327(261-401)$ & $p=0.01$ \\
\hline Lower lobe & $289(239-328)$ & $315(251-382)$ & $p=0.03$ \\
\hline Upper lobe & $359(331-421)$ & $376(290-451)$ & $\mathrm{p}=0.3$ \\
\hline \multicolumn{4}{|c|}{ ADA in $10^{4} \mu \mathrm{m}^{2}$ (IQR) } \\
\hline Total & $5.2(3.9-7.1)$ & $7.2(4.4-11.4)$ & $p=0.0002$ \\
\hline Lower lobe & $4.9(3.8-6.9)$ & $7.0(4.0-11.4)$ & $p=0.002$ \\
\hline Upper lobe & $6.7(4.1-7.5)$ & $8.6(6.0-11.7)$ & $\mathrm{p}=0.04$ \\
\hline \multicolumn{4}{|c|}{ EFT in $\mu \mathrm{m}$ (IQR) } \\
\hline Total & $6.3(5.2-7.7)$ & $6.4(5.5-7.7)$ & $p=0.2$ \\
\hline Lower lobe & $6.3(4.9-7.7)$ & $6.4(5.4-7.7)$ & $p=0.01$ \\
\hline Upper lobe & $6.2(5.4-6.8)$ & $6.4(5.6-7.6)$ & $\mathrm{p}=0.2$ \\
\hline \multicolumn{4}{|c|}{ Mean IQR per measurement } \\
\hline \multicolumn{4}{|c|}{ IQR LDAD in $\mu \mathrm{m}(95 \% \mathrm{Cl})$} \\
\hline Total & 97 (66 to 135$)$ & 135 (113 to 157$)$ & $p=0.07$ \\
\hline Lower lobe & 109 (61 to 156$)$ & 131 (106 to 156$)$ & $p=0.3$ \\
\hline Upper lobe & $115(68$ to 151$)$ & 142 (117 to 168$)$ & $p=0.04$ \\
\hline \multicolumn{4}{|c|}{ IQR ADA in $10^{4} \mu \mathrm{m}^{2}(95 \% \mathrm{Cl})$} \\
\hline Total & $2.9(1.9$ to 3.8$)$ & 5.1 (4.1 to 6.1$)$ & $\mathrm{p}=0.02$ \\
\hline Lower lobe & $3.8(2.6$ to 4.8$)$ & 4.5 (3.7 to 5.4$)$ & $\mathrm{p}=0.001$ \\
\hline Upper lobe & $2.3(1.4$ to 8.8$)$ & $5.5(4.1$ to 7.0$)$ & $\mathrm{p}=0.04$ \\
\hline \multicolumn{4}{|c|}{ IQR EFT in $\mu \mathrm{m}(95 \% \mathrm{Cl})$} \\
\hline Total & 2.7 (2.2 to 3.2$)$ & 2.5 (2.2 to 2.8$)$ & $\mathrm{p}=0.8$ \\
\hline Lower lobe & 2.8 (2.2 to 3.3$)$ & 2.6 (2.1 to 3.1$)$ & $\mathrm{p}=0.6$ \\
\hline Upper lobe & $1.6(1.2$ to 1.9$)$ & $2.2(1.9$ to 2.5$)$ & $p=0.03$ \\
\hline \multicolumn{4}{|c|}{ Autofluorescence intensity } \\
\hline \multicolumn{4}{|c|}{ mAFI (mean value $(95 \% \mathrm{Cl})$ ) } \\
\hline Total & $101(80$ to 121$)$ & 45 (33 to 58$)$ & $p<0.0001$ \\
\hline Lower lobe & & $46(31$ to 60$)$ & \\
\hline Upper lobe & & 45 (31 to 58$)$ & \\
\hline \multicolumn{4}{|c|}{ fAFI (mean value $(95 \% \mathrm{Cl}))$} \\
\hline Total & 79 (59 to 98$)$ & $29(17$ to 41$)$ & $\mathrm{p}<0.0001$ \\
\hline Lower lobe & & $34(21$ to 48$)$ & \\
\hline Upper lobe & & $37(24$ to 50$)$ & \\
\hline \multicolumn{4}{|c|}{ PA100 (mean \% (95\% Cl)) } \\
\hline Total & 45 (25 to 64$)$ & 73 (55 to 91$)$ & $\mathrm{p}=0.03$ \\
\hline Lower lobe & & 71 (55 to 86$)$ & \\
\hline Upper lobe & & 78 (61 to 96$)$ & \\
\hline CT density & & Emphysema & \\
\hline \multicolumn{4}{|c|}{ LMD (mean value HU $(95 \% \mathrm{Cl})$ ) } \\
\hline Total & & $-855(-922$ to -789$)$ & \\
\hline Lower lobe & & $-853(-881$ to -825$)$ & \\
\hline Upper lobe & & $-883(-916$ to -850$)$ & \\
\hline \multicolumn{4}{|c|}{ 15P (mean value $\mathrm{HU}(95 \% \mathrm{Cl}))$} \\
\hline Total & & $-970(-998$ to -941$)$ & \\
\hline Lower lobe & & -969 (-991 to -947$)$ & \\
\hline Upper lobe & & $-984(-1011$ to -957$)$ & \\
\hline \multicolumn{4}{|c|}{ LAA-950 (mean \% (95\% CI)) } \\
\hline Total & & 34 (20 to 48$)$ & \\
\hline Lower lobe & & $30(19$ to 40$)$ & \\
\hline Upper lobe & & 37 (26 to 48$)$ & \\
\hline
\end{tabular}

15P, the fifteenth percentile of pixel density; ADA, cross-sectional area of alveolar duct opening; EFT, elastic fibre thickness; fAFI, the autofluorescence intensity marking the fifteenth percentile; HU, Hounsfield units; LAA-950, the proportion of low attenuation areas with density less than -950 HU; LDAD, longest diameter of the alveolar openings at the level of the alveolar ducts; LMD, lung mean density; mAFI, median autofluorescence intensity; PA100, the proportional amount of pixels characterised by an autofluorescence intensity lower than 100. and FEV1\%pred $(\mathrm{r}=0.64, \mathrm{p}=0.01,95 \%$ CI 0.17 to 0.87 ) and between PA100 and residual volume $(\mathrm{RV}) /$ total lung capacity (TLC) $(\mathrm{r}=0.61, \mathrm{p}=0.02,95 \%$ CI 0.12 to 0.86). The IQR of ADA correlates well with RV/TLC ( $\mathrm{r}=0.67, \mathrm{p}=0.0009,95 \%$ CI 0.33 to 0.86 ).

\section{DISCUSSION}

pCLE can detect an increase in ADA and its variability as well as a regional decrease in AFI in emphysema. Both pCLE morphometry and AFI correlate with lung functional parameters. On a lobar level, the decrease in AFI correlates with a decrease in lobar attenuation on CT.

Recently, structure-function relationship in emphysema was demonstrated through the use of pCLE. ${ }^{3}$ The current study corroborates these morphometric data and suggests that the variability in dimensions of the alveolar duct openings increases in emphysema as compared with healthy subjects. Moreover, this variability correlates with RV/TLC, a lung functional parameter for hyperinflation, therefore endorsing the theoretical concept of the 'competition for space' in emphysema. ${ }^{4}$ Our morphometric data are somewhat comparable with studies of light microscopy and stereology in emphysema ${ }^{5}$ showing that destruction of the alveolar space is correlated with FEV1 and elastic recoil. ${ }^{6}$

Whether the reported morphological changes in elastic fibres and the associated changes in AFI are independent of hyperinflation and a true marker for elastic fibre disarrangement remains to be confirmed. Moreover, the analytical method used in this study is complex, time-consuming and difficult to reproduce.

pCLE imaging has a potential to deliver diagnostic information complementary to lung function and CT scanning, provided that it is able to gain diagnostic accuracy and that quantitative data can be obtained in a user-friendly way. As a result, computerised analysis and enhanced processing of endoscopic images will play an important role in future developments. There is a potential role for computerised digital frame stabilisation, noise reduction and pattern recognition before the current technique could be implemented as a diagnostic tool in clinical practice. ${ }^{7}$

Nevertheless, these structural findings and the assessment of heterogeneity in emphysema make in vivo pCLE imaging an interesting research tool, potentially playing its role as an endoscopic vector for the study of the regional microenvironment in emphysema. 


\section{J Yserbyt, C Dooms, W Janssens, G M Verleden}

Respiratory Division, Department of Clinical and Experimental Medicine, University Hospitals Leuven, KU Leuven, Belgium

Correspondence to Dr J Yserbyt, Respiratory Division, Department of Clinical and Experimental Medicine, University Hospitals Leuven, Herestraat 49, Leuven 3000, Belgium; Jonas.yserbyt@uzleuven.be

Contributors Substantial contributions to the conception or design of the work: JY, CD, WJ and GMV. Substantial contributions to the acquisition and analysis: JY. Substantial contributions to interpretation of data: JY, CD, WJ and GMV. Drafting of the work: JY. Substantial contributions to revising it critically for important intellectual content and final approval of the version to be published: JY, CD, WJ and GMV. Agreement to be accountable for all aspects of the work in ensuring that questions related to the accuracy or integrity of any part of the work are appropriately investigated and resolved: JY, CD, WJ and GMV.

Funding Financially supported by the Fonds voor Wetenschappelijk Onderzoek (FWO), Belgium.

Competing interests None declared.
Ethics approval Ethics Committee of the University Hospital Leuven.

Provenance and peer review Not commissioned; externally peer reviewed.

- Additional material is published online only. To view please visit the journal online (http://dx.doi.org/10. 1136/thoraxjnl-2016-209746)

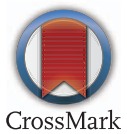

To cite Yserbyt J, Dooms C, Janssens W, et al. Thorax 2018;73:188-190.

Received 17 November 2016

Revised 15 March 2017

Accepted 27 March 2017

Published Online First 13 April 2017

Thorax 2018:73:188-190.

doi:10.1136/thoraxjnl-2016-209746

\section{REFERENCES}

1 Thiberville L, Moreno-Swirc S, Vercauteren T, et al. In Vivo imaging of the bronchial wall microstructure using fibered confocal fluorescence microscopy. Am J Respir Crit Care Med 2007;175:22-31.

2 Thiberville L, Salaün M, Lachkar $\mathrm{S}$, et al. Human in vivo fluorescence microimaging of the alveolar ducts and sacs during bronchoscopy. Eur Respir 2009;33:974-85.

3 Cosío B, Shafiek H, Fiorentino F, et al. Structurefunction relationship in COPD revisited : an in vivo microscopic view. Thorax 2014;69:724-30.

4 Verbeken EK, Cauberghs M, van de Woestijne K. Membranous bronchioles and connective tissue network of normal and emphysematous lungs. J Appl Physiol 1996;81:2468-80.

5 Mühlfeld C, Ochs M. Quantitative microscopy of the lung: a problem-based approach. Part 2: stereological parameters and study designs in various diseases of the respiratory tract. Am I Physiol Lung Cell Mol Physiol 2013:305:L205-21.

6 Saetta M, Shiner RJ, Angus GE, et al. Destructive index: a measurement of lung parenchymal destruction in smokers. Am Rev Respir Dis 1985;131:764-9

7 Perperidis A, Akram A, Altmann Y, et al. Automated detection of uninformative frames in pulmonary optical endomicroscopy. IEEE Trans Biomed Eng 2017;64:87-98 